\title{
Hecke operators type and generalized Apostol-Bernoulli polynomials
}

\author{
Aykut Ahmet Aygunes ${ }^{1}$, Abdelmejid Bayad² and Yilmaz Simsek ${ }^{1 *}$
}

\section{"Correspondence:}

ysimsek@akdeniz.edu.tr

'Department of Mathematics,

Faculty of Science, Akdeniz

University, Campus, Antalya, 07058,

Turkey

Full list of author information is

available at the end of the article

\begin{abstract}
In this paper, we construct some Hecke-type operators acting on the complex polynomials space, and we prove their commutativity. By means of this commutativity, we find a new approach to establish the generating function of the Apostol-Bernoulli type polynomials which are eigenfunctions of these Hecke-type operators. Moreover, we derive many useful identities related to these operators and polynomials.

MSC: $11 \mathrm{M} 35 ; 30 \mathrm{~B} 40 ; 30 \mathrm{~B} 50$

Keywords: Hecke operators; Hurwitz zeta function; generalized Apostol-Bernoulli type polynomials; Bernoulli polynomials; Euler polynomials
\end{abstract}

\section{Introduction}

The Hecke operators have many applications in various spaces like the space of elliptic modular forms, the space of polynomials and others. Many mathematicians applied them to obtain applications in analytic number theory, harmonic analysis, theoretical physics, equidistribution of Hecke points on a family of homogeneous varieties, and cohomology. For instance, Hecke operators are used to investigate and study Fourier coefficients of modular forms, to explore other properties of the Hecke-eigenforms, which satisfy many interesting arithmetic relations. For more details on Hecke operators, see [1, 2]. Recently, the Hurwitz zeta functions and the Apostol-Bernoulli polynomials have been studied by many authors, for example, see ( $c f$. [3-12], the others).

The main motivation of this paper is to introduce and study new Hecke-type operators on the ring of $\mathbb{C}[x]$. We study fundamental properties of these operators. We derive relations between these operators, the Hurwitz zeta functions and Apostol-Bernoulli type polynomials.

Our results are new and useful in applied mathematics and computation, analytic number theory and related areas. There are many reasons for being interested by Hecke-type operators. In particular, these operators are linear operators and are closely related to Raabe's multiplication theorem $[9,10]$. We recall the statement of this theorem, for any positive integer $m \geq 1$ we have

$$
\sum_{k=0}^{m-1} B_{n}\left(\frac{x+k}{m}\right)=m^{1-n} B_{n}(x), \quad \forall n \in \mathbb{N},
$$

() 2013 Aygunes et al.; licensee Springer. This is an Open Access article distributed under the terms of the Creative Commons Attribution License (http://creativecommons.org/licenses/by/2.0), which permits unrestricted use, distribution, and reproduction in any medium, provided the original work is properly cited. 
where $B_{n}(x)$ are the well-known Bernoulli polynomials. Conversely, from the paper [11] of Lehmer, it is well known that the Raabe's theorem gives a characterization of the Bernoulli polynomials. As an application, of the main result of this paper, the Lehmer's [11] approach will be generalized to the Apostol-Bernoulli type polynomials. These polynomials plays a central role in the computational number theory.

In order to state our results, we fix the following notations and definitions. Let $a, N$ be positive integers and $d \in \mathbb{C} \backslash\{0\}$ and $\xi_{N}$ be a primitive root of unity of order $N$. We consider the functions $\chi_{a, N}: \mathbb{N} \rightarrow \mathbb{C}$ given by

$$
\chi_{a, N}(k)= \begin{cases}\xi_{N}^{k}, & N \geq 2 \\ \frac{1}{a}, & N=1\end{cases}
$$

We define the partial Hecke-type operators associated to $\chi_{a, N}$ and $d$ as follows:

$$
T_{a, d, N}(P)(x):=\sum_{k=0}^{a-1} \chi_{a, N}(k) P\left(\frac{x+d k}{a}\right), \quad P(X) \in \mathbb{C}[X] .
$$

The total Hecke-type operators associated to $N$ and $d$ are defined by

$$
T_{d, N}:=\sum_{a \equiv 1(N)} T_{a, d, N}
$$

\section{Main results}

We have the following results.

Theorem 2.1 Let $a, N$ be positive integers and $d \in \mathbb{C} \backslash\{0\}$. Assume that $a \not \equiv 0(\bmod N)$. Then we have the following properties for the operators $T_{a, d, N}$ :

(i) The operator $T_{a, d, N}$ is linear and preserves the degree in $\mathbb{C}[x]$.

(ii) $\forall m \geq 1$,

$$
T_{a, d, N}\left(x^{m}\right)= \begin{cases}S_{d, 0}, & m=0 \\
a^{-m} x^{m}+a^{-m} \sum_{v=0}^{m-1}\left(\begin{array}{c}
m \\
v
\end{array}\right) S_{d, m-v}\left(\chi_{a, N}\right) \cdot x^{v}, & m \geq 1\end{cases}
$$

where

$$
S_{d, m-v}\left(\chi_{a, N}\right)=\sum_{k=0}^{a-1} \chi_{a, N}(k)(d k)^{m-v}= \begin{cases}\sum_{k=0}^{a-1} \frac{1}{a}(d k)^{m-v}, & N=1 ; \\ \sum_{k=0}^{a-1} \xi_{N}^{k}(d k)^{m-v}, & N \geq 2\end{cases}
$$

and $S_{d, 0}=S_{d, 0}\left(\chi_{a, N}\right)$.

Proof A simple computation gives the linearity of the operator $T_{a, d, N}$, so we omit it. Since $a \not \equiv 0(\bmod N)$, we can see easily that

$$
\sum_{k=0}^{a-1} \chi_{a, N}(k) \neq 0
$$


From the above equation, we obtain

$$
S_{d, 0} \neq 0 \text {. }
$$

Let us show how to compute $T_{a, d, N}\left(x^{m}\right)$. For $m=0$, we get

$$
T_{a, d, N}\left(x^{m}\right)=\sum_{k=0}^{a-1} \chi_{a, N}(k) .
$$

We end the proof by induction. Let $m \geq 1$, after an elementary manipulation we obtain

$$
T_{a, d, N}\left(x^{m}\right)=a^{-m} \sum_{v=0}^{m}\left\{\left(\begin{array}{c}
m \\
v
\end{array}\right) \sum_{k=0}^{a-1} \chi_{a, N}(k)(d k)^{m-v}\right\} x^{v}
$$

and, therefore, (ii) is satisfied.

We consider the restriction of the partial Hecke operator to the finite dimensional space

$$
\mathbb{C}_{m}[x]=\{P(x) \in \mathbb{C}[x]: \text { degree of } P(x) \leq m\} .
$$

By writing the operator $T_{a, d, N}$ in the canonical basis $\beta_{m}=\left(1, x, x^{2}, \ldots, x^{m}\right)$, and from (ii), we get the corresponding matrix. Using linear algebra, we will see that this matrix representation is useful and gives interesting results.

Proposition 2.2 For any $m \in \mathbb{N}$, let $\beta_{m}=\left(1, x, x^{2}, \ldots, x^{m}\right)$ be the canonical $\mathbb{C}$-basis of $\mathbb{C}_{m}[x]$. Then the matrix $M_{\beta_{m}}\left(T_{a, d, N}\right)$ corresponding to the operator $T_{a, d, N}$ (restricted to $\mathbb{C}_{m}[x]$ ) in the basis $\beta_{m}$ is given by

$$
M_{\beta_{m}}\left(T_{a, d, N}\right)=\left(\begin{array}{ccccc}
S_{d, 0} & a^{-1} S_{d, 1} & a^{-2} S_{d, 2} & \ldots & a^{-m} S_{d, m} \\
0 & a^{-1} S_{d, 0} & 2 a^{-2} S_{d, 1} & \ldots & a^{-m}\left(\begin{array}{c}
m \\
1
\end{array}\right) S_{d, m-1} \\
0 & 0 & a^{-2} S_{d, 0} & \ldots & a^{-m}\left(\begin{array}{c}
m \\
2
\end{array}\right) S_{d, m-2} \\
0 & 0 & 0 & \ldots & a^{-m}\left(\begin{array}{c}
m \\
3
\end{array}\right) S_{d, m-3} \\
\vdots & \vdots & \vdots & & \vdots \\
0 & 0 & 0 & \ldots & a^{-m} S_{d, 0}
\end{array}\right) .
$$

Remark 2.3 For any positive integer $a \geq 2$, the eigenvalues $S_{d, 0}, a^{-1} S_{d, 0}, a^{-2} S_{d, 0}, \ldots$, $a^{-m} S_{d, 0}$ of the matrix (1) are distinct. Then from the theory of linear algebra we deduce that the matrix (1) is a diagonalizable. Again, thanks to linear algebra, we know that there exists a sequence of polynomials $\left(P_{n, d, N}\right)_{n \in \mathbb{N}}$, which is a sequence of eigenpolynomials of (1). For more details, see the next section.

Theorem 2.4 The operators $T_{a, d, N}$ and $T_{b, d, N}$ commute if $a \equiv b \equiv 1(\bmod N)$.

Proof We consider the linear operators $T_{a, d, N}$ and $T_{b, d, N}$ and we must show that

$$
T_{a, d, N} T_{b, d, N}=T_{b, d, N} T_{a, d, N}
$$


for all $a \equiv 1(\bmod N)$. This equality is obvious when $N=1$. For $N \geq 2$, we have the following equalities:

$$
\left(T_{a, d, N} T_{b, d, N}\right)(P(x))=T_{a, d, N}\left(T_{b, d, N}(P(x))\right)=T_{a, d, N}\left(\sum_{k_{1}=0}^{b-1} \xi_{N}^{k_{1}} P\left(\frac{x+d k_{1}}{b}\right)\right) .
$$

The linearity of the of the operator $T_{a, d, N}$ implies that

$$
\left(T_{a, d, N} T_{b, d, N}\right)(P(x))=\sum_{k_{1}=0}^{b-1} \xi_{N}^{k_{1}} T_{a, d, N}\left(P\left(\frac{x+d k_{1}}{b}\right)\right)=\sum_{k_{1}=0}^{b-1} \xi_{N}^{k_{1}} \sum_{k_{2}=0}^{a-1} \xi_{N}^{k_{2}} P\left(\frac{\frac{x+d k_{1}}{b}+d k_{2}}{a}\right) .
$$

Then we deduce

$$
\left(T_{a, d, N} T_{b, d, N}\right)(P(x))=\sum_{k_{1}=0}^{b-1} \sum_{k_{2}=0}^{a-1} \xi_{N}^{k_{1}} \xi_{N}^{k_{2}} P\left(\frac{x+d\left(k_{1}+b k_{2}\right)}{a b}\right) .
$$

By setting $k=k_{1}+b k_{2}$, we obtain

$$
\left(T_{a, d, N} T_{b, d, N}\right)(P(x))=\sum_{k=0}^{a b-1} \xi_{N}^{k} P\left(\frac{x+d k}{a b}\right)=T_{a b, d, N}(P(x))=T_{b a, d, N}(P(x)) .
$$

Finally, we get our desired equality

$$
\left(T_{a, d, N} T_{b, d, N}\right)(P(x))=\left(T_{b, d, N} T_{a, d, N}\right)(P(x)) .
$$

\section{New characterization of Apostol-Bernoulli type polynomials}

As an application of our main results, we study the polynomials $P \in \mathbb{C}[x]$ satisfying the functional equation

$$
T_{a, d, N}(P)=a^{-n} P(x)
$$

where $a \equiv 1(N)$ and fixed integer $n \geq 1$.

Theorem 3.1 Let $a, N$ be positive integers and $d \in \mathbb{C} \backslash\{0\}$ such that $a \equiv 1(N)$. Then we have the following properties:

(i) There exists an unique sequence of monic polynomials $P_{n, d, N} \in \mathbb{C}[x]$ with $\operatorname{deg} P_{n, d, N}=n$ such that

$$
T_{a, d, N}\left(P_{n, d, N}\right)=a^{-n} P_{n, d, N} .
$$

(ii) Polynomials $P_{n, d, N}(x)$ are eigenfunctions for the operators $T_{n, d, N}$ with eigenvalues $N^{-n} \zeta\left(n, \frac{1}{N}\right)$, that is

$$
T_{d, N}\left(P_{n, d, N}\right)(x)=N^{-n} \zeta\left(n, \frac{1}{N}\right) P_{n, d, N}(x)
$$

where $\zeta(s, x)=\sum_{k \geq 0} \frac{1}{(x+k)^{s}}$ is the Hurwitz zeta function. 
Proof The existence of a sequence of monic polynomials $P$ is satisfied from Theorem 2.1 and Theorem 2.4.

Now we must observe the uniqueness of $\left(P_{n, d, N}\right)_{n \in \mathbb{N}}$. For this end, we take two different monic polynomials $P_{n, d, N}$ and $R_{n, d, N}$ of degree $n$ satisfying (2).

Suppose that $P_{n, d, N}(x)-R_{n, d, N}(x)=\Delta_{m}(x)=A_{0} x^{m}+A_{1} x^{m-1}+\cdots$, where $1 \leq m<n$ and $A_{0} \neq 0$. From (2) and the definition of $T_{a, d, N}$, we can write

$$
\sum_{k=0}^{a-1} \chi_{a, N}(k) P_{n, d, N}\left(\frac{x+d k}{a}\right)=a^{-n} P_{n, d, N}(x)
$$

and

$$
\sum_{k=0}^{a-1} \chi_{a, N}(k) R_{n, d, N}\left(\frac{x+d k}{a}\right)=a^{-n} R_{n, d, N}(x) .
$$

Subtracting (4) from (3), we get

$$
\sum_{k=0}^{a-1} \chi_{a, N}(k) \Delta_{m}\left(\frac{x+d k}{a}\right)=a^{-n}\left(A_{0} x^{m}+A_{1} x^{m-1}+\cdots\right) .
$$

Identifying the coefficients of $x^{m}$ on both sides, we have $A_{0}=a^{-n} A_{0}$, but this contradicts our stipulations that $A_{0} \neq 0, m<n$, and $a \geq 2$. Hence, the proof of (i) is completed.

We prove (ii). It is easy to see that

$$
T_{d, N}\left(P_{n, d, N}\right)(x)=\sum_{\substack{a \equiv 1(N) \\ a \geq 0}} T_{a, d, N}\left(P_{n, d, N}\right)(x)=\left(\sum_{\substack{a \equiv 1(N) \\ a \geq 0}} a^{-n}\right) P_{n, d, N}(x)
$$

and putting $a=1+k N$, we obtain

$$
T_{d, N}\left(P_{n, d, N}\right)(x)=\sum_{k \geq 0}(1+k N)^{-n} P_{n, d, N}(x)=N^{-n} \zeta\left(n, \frac{1}{N}\right) \cdot P_{n, d, N}(x) .
$$

Thanks to Theorem 2.4, we find the generating function of polynomials $\left(P_{n, d, N}\right)_{n \in \mathbb{N}}$ satisfying (2). More precisely, we have the following theorem.

Theorem 3.2 For all $n \geq 1$, we have the following results:

(i) $\frac{d}{d x} P_{n, d, N}(x)=n P_{n-1, d, N}(x)$.

(ii) $P_{n, d, N}(d)=\xi_{N}^{-1} P_{n, d, N}(0)$.

(iii) The difference formula of $\left(P_{n, d, N}\right)_{n \in \mathbb{N}}$ is given by

$$
P_{n, d, N}(x+d)-\xi_{N}^{-1} P_{n, d, N}(x)= \begin{cases}n x^{n-1}, & N=1 ; \\ \left(1-\xi_{N}^{-1}\right) x^{n}, & N \geq 2 .\end{cases}
$$

Proof We prove (i). From (2) and the definition of the operators $T_{a, d, N}$, we have

$$
\sum_{k=0}^{a-1} \chi_{a, N}(k) P_{n, d, N}\left(\frac{x+d k}{a}\right)=a^{-n} P_{n, d, N}(x),
$$


we derive this equation and obtain

$$
\sum_{k=0}^{a-1} \chi_{a, N}(k) \frac{P_{n, d, N}^{\prime}\left(\frac{x+d k}{a}\right)}{n}=a^{1-n} \frac{P_{n, d, N}^{\prime}(x)}{n} .
$$

Since $\frac{P_{n, d, N}^{\prime}(x)}{n}$ is monic with degree $n-1$, from Theorem 3.1(i), we arrive at

$$
\frac{P_{n, d, N}^{\prime}(x)}{n}=P_{n-1, d, N}(x)
$$

We prove (ii). For $N \geq 2$, by taking $\chi_{a, N}(k)=\xi_{N}^{k}$ and $a=N+1$, we have

$$
\sum_{k=0}^{N} \xi_{N}^{k} P_{n, d, N}\left(\frac{x+d k}{N+1}\right)=(N+1)^{-n} P_{n, d, N}(x) .
$$

In the above equation, putting $x=0$ and $x=d$, respectively, we arrive at

$$
\sum_{k=0}^{N} \xi_{N}^{k} P_{n, d, N}\left(\frac{d k}{N+1}\right)=(N+1)^{-n} P_{n, d, N}(0)
$$

and

$$
\sum_{k=0}^{N} \xi_{N}^{k} P_{n, d, N}\left(\frac{d(k+1)}{N+1}\right)=(N+1)^{-n} P_{n, d, N}(d) .
$$

Multiplying each side of (7) by $\xi_{N}$ and then substrate it from (6), we have the following relation:

$$
P_{n, d, N}(0)-\xi_{N}^{N+1} P_{n, d, N}(d)=(N+1)^{-n}\left(P_{n, d, N}(0)-\xi_{N} P_{n, d, N}(d)\right) .
$$

Since $\xi_{N}^{N+1}=\xi_{N}$, we obtain that $P_{n, d, N}(d)=\xi_{N}^{-1} P_{n, d, N}(0)$ for all $n \geq 1$ and $N \geq 2$.

We prove (iii). We can write

$$
P_{n, d, N}(x)=\sum_{k=0}^{n} P_{n, d, N}^{(k)}(0) \frac{x^{k}}{k !}, \quad P_{n, d, N}(x+d)=\sum_{k=0}^{n} P_{n, d, N}^{(k)}(d) \frac{x^{k}}{k !} .
$$

On the other hand, by using Theorem 3.2(i), we get

$$
\begin{aligned}
& P_{n, d, N}(x)=\sum_{k=0}^{n}\left(\begin{array}{l}
n \\
k
\end{array}\right) P_{n-k, d, N}(0) x^{k}, \\
& P_{n, d, N}(x+d)=\sum_{k=0}^{n}\left(\begin{array}{l}
n \\
k
\end{array}\right) P_{n-k, d, N}(d) x^{k} .
\end{aligned}
$$

We multiply the each side of (9) by $\xi_{N}$ and then substrate (8) from (9), we arrive to

$$
\xi_{N} P_{n, d, N}(x+d)-P_{n, d, N}(x)=\sum_{k=0}^{n}\left(\begin{array}{l}
n \\
k
\end{array}\right)\left(P_{n-k, d, N}(d)-\xi_{N} P_{n-k, d, N}(0)\right) x^{k} .
$$


From Theorem 3.2(ii) and equality $P_{0, d, N}(d)=P_{0, d, N}(d)=1$, we get

$$
\xi_{N} P_{n, d, N}(x+d)-P_{n, d, N}(x)=-\left(\left(\begin{array}{l}
n \\
n
\end{array}\right) P_{0, d, N}(0)-\left(\begin{array}{l}
n \\
n
\end{array}\right) \xi_{N} P_{0, d, N}(1)\right) x^{n}=\left(\xi_{N}-1\right) x^{n}
$$

Therefore, we obtain the desired result.

Using Theorem 2.4 and Theorem 3.1, we can establish the following result.

Theorem 3.3 For $a \equiv 1(N)$, the generating function of $\left(P_{n, d, N}\right)_{n \in \mathbb{N}}$ is given by

$$
F_{d, N}(x, t)= \begin{cases}\frac{d t e^{x t}}{e^{d t}-1}, & \text { if } N=1 \\ \frac{\left(\xi_{N}-1\right) e^{x t}}{\xi_{N} e^{d t}-1}, & \text { if } N \geq 2 .\end{cases}
$$

Proof Let $N \geq 2$ integer and write

$$
\frac{\left(\xi_{N}-1\right) e^{x t}}{\xi_{N} e^{d t}-1}=\sum_{n \geq 0} Q_{n, d}\left(x, \xi_{N}\right) \frac{t^{n}}{n !}
$$

Using the difference formula in Theorem 3.2, we get

$$
\xi_{N}^{m-1} P_{n, d, N}(m d)-\xi_{N}^{-1} P_{n, d, N}(0)=\left(1-\xi_{N}^{-1}\right) \sum_{j=0}^{m-1} \xi_{N}^{j}(j d)^{n}
$$

We consider the generating function

$$
\begin{aligned}
\sum_{n \geq 0}\left(\xi_{N}^{m} P_{n, d, N}(m d)-P_{n, d, N}(0)\right) \frac{t^{n}}{n !} & =\left(\xi_{N}-1\right) \sum_{j=0}^{m-1} \xi_{N}^{j} \sum_{n \geq 0} \frac{(j d t)^{n}}{n !} \\
& =\left(\xi_{N}-1\right) \frac{\xi_{N}^{m} e^{m d t}-1}{\xi_{N} e^{d t}-1} \\
& =\xi_{N}^{m} \frac{\left(\xi_{N}-1\right) e^{m d t}}{\xi_{N} e^{d t}-1}-\frac{\left(\xi_{N}-1\right)}{\xi_{N} e^{d t}-1} \\
& =\xi_{N}^{m} \sum_{n \geq 0} Q_{n, d}\left(m d, \xi_{N}\right) \frac{t^{n}}{n !}-\sum_{n \geq 0} Q_{n, d}\left(0, \xi_{N}\right) \frac{t^{n}}{n !}
\end{aligned}
$$

thus,

$$
\sum_{n \geq 0}\left(\xi_{N}^{m} P_{n, d, N}(m d)-P_{n, d, N}(0)\right) \frac{t^{n}}{n !}=\sum_{n \geq 0}\left\{\xi_{N}^{m} Q_{n, d}\left(m d, \xi_{N}\right)-Q_{n, d}\left(0, \xi_{N}\right)\right\} \frac{t^{n}}{n !}
$$

We compare the coefficients of $x^{n}$ in the above equation and we obtain

$$
\xi_{N}^{m} P_{n, d, N}(m d)-P_{n, d, N}\left(0, \xi_{N}\right)=\xi_{N}^{m} Q_{n, d}\left(m d, \xi_{N}\right)-Q_{n, d}\left(0, \xi_{N}\right)
$$

In particular, if we take $m \equiv 0(N)$, then we have

$$
P_{n, d, N}(m d)-P_{n, d, N}\left(0, \xi_{N}\right)=Q_{n, d}\left(m d, \xi_{N}\right)-Q_{n, d}\left(0, \xi_{N}\right)
$$


Therefore, we note that the polynomials $P_{n, d, N}(x)-P_{n, d, N}\left(0, \xi_{N}\right)$ and $Q_{n, d}\left(x, \xi_{N}\right)-Q_{n, d}\left(0, \xi_{N}\right)$ are equal on the infinite set $\{x=m d$ : with $m \equiv 0(\bmod N)\}$. Then we can write for all $x \in \mathbb{C}$,

$$
P_{n, d, N}(x)-P_{n, d, N}(0)=Q_{n, d}\left(x, \xi_{N}\right)-Q_{n, d}\left(0, \xi_{N}\right)
$$

Now, by derivation on $x$ we get

$$
n P_{n-1, d, N}(x)=P_{n, d, N}^{\prime}(x)=Q_{n, d, N}^{\prime}(x d)=n Q_{n-1, d, N}(x) .
$$

We obtain the equality

$$
P_{n, d, N}(x)=Q_{n, d, N}(x) .
$$

Hence, we obtain the generating function of $P_{n, d, N}$.

Remark 3.4 The case $d=1$ of Theorem 3.3 recovers the so-called generalized Bernoulli and Euler polynomials, which are studied in [9].

\section{Eigenpolynomials attached to Dirichlet characters}

Let $d$ be a positive integer, $\psi$ be a Dirichlet character modulo $d$. We associate to $\psi, d, N$ the polynomials $P_{n, \psi, d}\left(x, \xi_{N}\right)$ defined by the generating function

$$
\sum_{n \geq 0} P_{n, \psi, d}\left(x, \xi_{N}\right) \frac{t^{n}}{n !}= \begin{cases}\left(\xi_{N}-1\right) \sum_{b=1}^{d} \frac{\psi(b) e^{(x+b) t}}{\xi_{N} e^{d t}-1}, & N \geq 2 \\ \sum_{b=1}^{d} \frac{\psi(b) t e^{(x+b) t}}{e^{d t}-1}, & N=1 .\end{cases}
$$

Then we have the interesting relations.

Theorem 4.1 Let $d$ be a positive integer, $\psi$ be a Dirichlet character modulo $d$. Then we have the identity

$$
P_{n, \psi, d}\left(x, \xi_{N}\right)=\sum_{b=1}^{d} \psi(b) P_{n, d, N}(x+b)
$$

which is equivalent to the following equality:

$$
P_{n, \psi, d}\left(x, \xi_{N}\right)=\sum_{k=0}^{n}\left(\begin{array}{l}
n \\
k
\end{array}\right) \sum_{b=1}^{d} \psi(b) P_{n-k, d, N}(x) b^{k}
$$

Proof The proof for $N=1$ is trivial, we omit it. For $N \geq 2$, by using equation (10), we obtain

$$
\sum_{n \geq 0} P_{n, \psi, d}\left(x, \xi_{N}\right) \frac{t^{n}}{n !}=\sum_{b=1}^{d} \frac{\left(\xi_{N}-1\right) \psi(b) e^{(x+b) t}}{\xi_{N} e^{d t}-1}=\sum_{b=1}^{d} \psi(b) \sum_{n \geq 0} P_{n, d, N}(x+b) \frac{t^{n}}{n !} .
$$

Taking the coefficients of $\frac{t^{n}}{n !}$ in the left and right sides of above equation, we have

$$
P_{n, \psi, d}\left(x, \xi_{N}\right)=\sum_{b=1}^{d} \psi(b) P_{n, d, N}(x+b) .
$$


Now we prove (ii). By equation (10) we have

$$
\begin{aligned}
\sum_{n \geq 0} P_{n, \psi, d}\left(x, \xi_{N}\right) \frac{t^{n}}{n !} & =\sum_{b=1}^{d} \frac{\left(\xi_{N}-1\right) \psi(b) e^{(x+b) t}}{\xi_{N} e^{d t}-1} \\
& =\frac{\left(\xi_{N}-1\right) e^{x t}}{\xi_{N} e^{d t}-1} \sum_{b=1}^{d} \psi(b) e^{b t} \\
& =\left(\sum_{n \geq 0} P_{n, d, N}(x) \frac{t^{n}}{n !}\right)\left(\sum_{n \geq 0} \sum_{b=1}^{d} \psi(b) b^{n} \frac{t^{n}}{n !}\right)
\end{aligned}
$$

then we have

$$
\sum_{n \geq 0} P_{n, \psi, d}\left(x, \xi_{N}\right) \frac{t^{n}}{n !}=\sum_{n \geq 0}\left(\sum_{k=0}^{n}\left(\begin{array}{l}
n \\
k
\end{array}\right) \sum_{b=1}^{d} \psi(b) P_{n-k, d, N}(x) b^{k}\right) \frac{t^{n}}{n !} .
$$

Comparing the coefficients of $\frac{t^{n}}{n !}$ in both sides in the last equality, we obtain the desired result.

Theorem 4.2 For any positive integers $N$ and a such that $a \equiv 1(N)$. Then the polynomials $P_{n, \psi, d}$ are eigenpolynomials for Hecke type operators $T_{a, d, N}$.

Proof From Theorem 3.1, we have

$$
T_{a, d, N}\left(P_{n, d, N}(x)\right)=a^{-n} P_{n, d, N}(x),
$$

then for any integer $b$ we have

$$
T_{a, d, N}\left(P_{n, d, N}(x+b)\right) \psi(b)=a^{-n} P_{n, d, N}(x+b) \psi(b) .
$$

Summing over all $1 \leq b \leq d$

$$
\sum_{b=1}^{d} \psi(b) T_{a, d, N}\left(P_{n, d, N}(x+b)\right)=a^{-n} \sum_{b=1}^{d} \psi(b) P_{n, d, N}(x+b) .
$$

Therefore, by linearity of the Hecke operator we obtain

$$
T_{a, d, N}\left(P_{n, \chi, d}\left(x, \xi_{N}\right)\right)=T_{a, d, N}\left(\sum_{b=1}^{d} \chi(b) P_{n, d, N}(x+b)\right)=\sum_{b=1}^{d} \chi(b) T_{a, d, N}\left(P_{n, d, N}(x+b)\right) .
$$

We then obtain our formula

$$
T_{a, d, N}\left(P_{n, \chi, d}\left(x, \xi_{N}\right)\right)=a^{-n} \sum_{b=1}^{d} \chi(b) P_{n, d, N}(x+b)=a^{-n} P_{n, \chi, d}\left(x, \xi_{N}\right) .
$$

Theorem 4.3 For all integer $n \geq 1$, the difference formula of $\left(P_{n, \psi, d}\right)\left(x, \xi_{N}\right)$ is given by

$$
P_{n, \psi, d}\left(x+d, \xi_{N}\right)-\xi_{N}^{-1} P_{n, \psi, d}\left(x, \xi_{N}\right)= \begin{cases}n \sum_{b=1}^{d} \psi(b)(x+b)^{n-1}, & N=1 \\ \left(1-\xi_{N}^{-1}\right) \sum_{b=1}^{d} \psi(b)(x+b)^{n}, & N \geq 2\end{cases}
$$


Proof From Theorem 3.2, we know that, for all $n \geq 1$,

$$
P_{n, d, N}(x+d)-\xi_{N}^{-1} P_{n, d, N}(x)= \begin{cases}n x^{n-1}, & N=1 \\ \left(1-\xi_{N}^{-1}\right) x^{n}, & N \geq 2\end{cases}
$$

Let $N \geq 2$. By using Theorem 4.1, we get

$$
P_{n, \psi, d}\left(x+d, \xi_{N}\right)=\sum_{b=1}^{d} \psi(b) P_{n, d, N}(x+b+d)
$$

and

$$
-P_{n, \psi, d}\left(x, \xi_{N}\right)=-\sum_{b=1}^{d} \psi(b) P_{n, d, N}(x+b) .
$$

Therefore,

$$
\begin{aligned}
P_{n, \psi, d}\left(x+d, \xi_{N}\right)-\xi_{N}^{-1} P_{n, \psi, d}\left(x, \xi_{N}\right) & =\sum_{b=1}^{d} \psi(b)\left(P_{n, d, N}(x+b+d)-\xi_{N}^{-1} P_{n, d, N}(x+b)\right) \\
& =\left(1-\xi_{N}^{-1}\right) \sum_{b=1}^{d} \psi(b)(x+b)^{n} .
\end{aligned}
$$

Let $N=1$. By using Theorem 4.1, we get

$$
P_{n, \psi, d}\left(x+d, \xi_{N}\right)=\sum_{b=1}^{d} \psi(b) P_{n, d, N}(x+b+d)
$$

and

$$
-P_{n, \psi, d}\left(x, \xi_{N}\right)=-\sum_{b=1}^{d} \psi(b) P_{n, d, N}(x+b) .
$$

Therefore,

$$
\begin{aligned}
P_{n, \psi, d}\left(x+d, \xi_{N}\right)-\xi_{N}^{-1} P_{n, \psi, d}\left(x, \xi_{N}\right) & =\sum_{b=1}^{d} \psi(b)\left(P_{n, d, N}(x+b+d)-P_{n, d, N}(x+b)\right) \\
& =n \sum_{b=1}^{d} \psi(b)(x+b)^{n-1} .
\end{aligned}
$$


Author details

${ }^{1}$ Department of Mathematics, Faculty of Science, Akdeniz University, Campus, Antalya, 07058, Turkey. ${ }^{2}$ Departement de mathematiques, Universite d'Evry Val d'Essonne, Batiment I.B.G.B.I., 3eme etage, 23 Bd. de France, Evry Cedex, 91037,

France.

\section{Acknowledgements}

Dedicated to Professor Hari M Srivastava.

All authors are partially supported by Research Project Offices Akdeniz Universities. We would like to thank for referees for their valuable comments.

\section{Received: 24 January 2013 Accepted: 26 March 2013 Published: 10 April 2013}

\section{References}

1. Serre, J-P: Cours D'arithmétique. PUF, Paris (1970)

2. Hellegouarch, Y: Invitation aux Mathématiques de Fermat-Wiles. Dunod, Paris (2001)

3. Kim, T: Note on the Euler numbers and polynomials. Adv. Stud. Contemp. Math. 17(2), 131-136 (2008)

4. Kim, T: Symmetry of power sum polynomials and multivariate fermionic $p$-adic invariant integral on $\mathbb{Z}_{p}$. Russ. J. Math. Phys. 16(1), 93-96 (2009)

5. Ozden, H, Simsek, Y, Srivastava, HM: A unified presentation of the generating functions of the generalized Bernoulli, Euler and Genocchi polynomials. Comput. Math. Appl. 60, 2779-2787 (2010)

6. Srivastava, HM, Ozarslan, MA, Kaanoglu, C: Some generalized Lagrange-based Apostol-Bernoulli, Apostol-Euler and Apostol-Genocchi polynomials. Russ. J. Math. Phys. 20, 110-120 (2013)

7. Srivastava, HM, Choi, J: Zeta and q-Zeta Functions and Associated Series and Integrals. Elsevier, Amsterdam (2012)

8. Srivastava, HM: Some generalizations and basic (or $q$-) extensions of the Bernoulli, Euler and Genocchi polynomials. Appl. Math. Inf. Sci. 5, 390-444 (2011)

9. Bayad, A, Aygunes, AA, Simsek, Y: Hecke operators and generalized Bernoulli-Euler polynomials. J. Algebra Number Theory, Adv. Appl. 3, 111-122 (2010)

10. Raabe, JL: Zurückführung einiger summen and bestimmten integrale auf die Jacob Bernoullische function. J. Reine Angew. Math. 42, 348-376 (1851)

11. Lehmer, DH: A new approach to Bernoulli polynomials. Am. Math. Mon. 95, 905-911 (1998)

12. Kim, T: Symmetry identities for the twisted generalized Euler polynomials. Adv. Stud. Contemp. Math. 19, 111-118 (2009)

doi:10.1186/1687-1812-2013-92

Cite this article as: Aygunes et al.: Hecke operators type and generalized Apostol-Bernoulli polynomials. Fixed Point Theory and Applications 2013 2013:92.

\section{Submit your manuscript to a SpringerOpen ${ }^{\ominus}$ journal and benefit from:}

- Convenient online submission

- Rigorous peer review

- Immediate publication on acceptance

- Open access: articles freely available online

- High visibility within the field

- Retaining the copyright to your article 